\title{
Streamlining Onion Pungency Analyses
}

\section{William M. Randle and Mark L. Bussard \\ Department of Horticulture, University of Georgia, Athens, GA 30602}

Additional index words. Allium cepa, breeding, flavor, pyruvic acid

Breeding onions for desired pungency requires reliable methods for discriminating between individuals in a segregating population. Organoleptic evaluation has been used traditionally in onion breeding programs; however, it lacks precision and repeatability, especially when evaluating highly pungent onions. Schwimmer and Weston's method (1961) has been proven to be effective in quantifying onion pungency (Lancaster and Boland, 1990; Whitaker, 1976). Pungency and other flavors are formed from the hydrolysis of S-alk(en)yl cysteine sulfoxide precursor molecules by alliinase following cell disruption. This cleavage produces S-alk(en) sulfenic acid, pyruvic acid, and ammonia. Schwimmer and Weston's method quantifies pyruvic acid formation from the enzymatic cleavage of the flavor precursor molecules. Pyruvic acid reacts with 2,4-dinitrophenyl hydrazine to produce a colored derivative, which can be measured spectrophotometrically. Although pyruvic acid is a relatively stable compound in this reaction, it is also a common metabolic product in the plant that can upwardly bias enzymatic hydrolysis measurements. As a result, pyruvic acid background levels are determined by thermal deactivation of the enzyme system before analysis. To determine the amount of pyruvic acid formed enzymatitally, the background concentration is subtracted from total pyruvic acid. Schwimmer and Weston's method, however, is time-consuming and cumbersome, which makes routine and systematic evaluation of breeding material and experimental treatments costly. We propose a routine method for evaluating onion pungency that reduces time and costs.

Processing a single bulb in our laboratory using Schwimmer and Weston's method took $\approx 74 \mathrm{~min}-58 \mathrm{~min}$ to extract and purify the onion juice for total and background measurements, and $16 \mathrm{~min}$ to quantify pyruvic acid. To shorten the time required to extract and purify the juice, we designed and devel-

Received for publication 16 Apr. 1992. Accepted for publication 10 Sept. 1992. The cost ofpublishing this paper was defrayed in part by the payment of page charges. Under postal regulations, this paper therefore must be hereby marked advertisement solely to indicate this fact. oped a press to squeeze onion juice from the bulb that was free of solid particles. The raw juice was then used for pyruvic acid measurements. In contrast, Schwimmer and Weston's method requires grinding, centrifuging, and filtering the juice to obtain sufficiently pure levels.

The press is driven by a pneumatic piston that crushes an onion placed in a barrel slightly larger than the piston. Two stainless steel screens (4- and 1-mm mesh) are above a press plate, at the bottom of the barrel. An angled groove is just outside the press plate to channel liquid away from the onion as it is pressed. Thus, the nonsoluble solids of the bulb are retained while the juice escapes to a holding receptacle. Extracting juice from a bulb took $\approx 3 \mathrm{~min} / \mathrm{sample}$.

We compared the purity of the raw juice obtained from the press with that obtained using Schwimmer and Weston's method. A white fresh onion and a yellow storage onion were purchased at a local market. Eight bulbs of each type were selected and halved longitudinally for comparing juice extraction methods. Either pressed raw juice or pressed juice filtered through no. 42 Whatman paper or centrifuged at $10,000 \times g$ for $9 \mathrm{~min}$ and then filtered through no. 42 paper was analyzed. Pyruvic acid was quantified according to Schwimmer and Weston (1961). Each comparison was repeated at least three times, and data were subjected to paired $t$ tests. No differences were found among the standard methodology and raw, filtered, or centrifuged and filtered juice from the press for the white (6.2, 6.1, 6.1, and $6.1 \mu \mathrm{mol}$ pyruvic acid $/ \mathrm{ml}$ juice, respectively) and yellow onion (10.3, $10.2,10.2,10.2 \mu \mathrm{mol}$ pyruvic acid/ml juice, respectively). The correlation between raw juice and the standard method for pyruvic acid development from individual bulbs was $r^{2}=$ 0.99 .

Occasionally, a bulb would disintegrate or explode onto the press plate from the pressure of the piston, resulting in solid material in the juice. Filtering once through no. 42 paper sufficiently removed solid particles from the juice. Placing the bulb's cut side toward the press plate was the best method for juice extraction. Whole bulbs could be pressed if they were small enough to fit into the barrel.
We modified Schwimmer and Weston's method further by attaching a diverter to the draining channel of the press plate assembly. The diverter split the juice stream in half; thus, total and background pyruvic acid concentrations were determined from a single pressing. One half flowed into a beaker where pyruvic acid was allowed to form. The other half flowed into a preweighed beaker containing $2.5 \%$ trichlorolacetic acid (TCA) to deactivate the juice enzymes for background pyruvic acid measurement. This ratio was sufficient to deactivate enzymes in the flavorreaction. After collecting the juice, the beaker was agitated and reweighed, and enough $2.5 \%$ TCA was added after $25 \%(\mathrm{w} / \mathrm{v})$ of the bulb was pressed for a 1 juice : 1 TCA (w/v) volume. In a study repeated three times using eight halved yellow storage onions, no significant difference existed between the TCA method described above and the standard Schwimmer and Weston method for determining background pyruvic acid concentrations. Background pyruvic acid concentration means for the TCA and standard methods were 3.7 and $3.6 \mu \mathrm{mol} \cdot \mathrm{g}^{-1}$ fresh weight, respectively.

Because running a sample takes less time with the press, more samples can be run per unit time, thereby reducing the cost per sample. Using our method, several onion samples can be analyzed simultaneously, thereby allowing a single person to process up to 15 bulbs per hour. Only 36 bulbs could be analyzed per 8 $h$ day in our laboratory using the standard method and running six bulbs simultaneously. Clean-up time and the cost of cleaning glassware was also significantly reduced, since $75 \%$ less glassware was used with the new method.

In conclusion, no significant difference existed in the accuracy of pyruvic acid measurements made by our method and Schwimmer and Weston's Our method decreased the time required to process a single bulb from 78 (standard method) to $19 \mathrm{~min}$, and thereby increased the likelihood that chemical analysis can be used in onion breeding programs when selecting for pungency. Although we have described a specific press, any system that rapidly extracts relatively pure onion juice from bulb tissue has potential for assessing pungency levels quickly.

\section{Literature Cited}

Lancaster, J.E. and M.J. Boland. 1990. Flavor biochemistry, p. 33-72. In: H.D. Rabinowitch and J.L. Brewster (eds.). Onions and allied crops. vol. 3. CRC Press, Boca Raton, Fla.

Schwimmer, S. and W.J. Weston. 1961. Enzymatic development of pyruvic acid as a measure of pungency. J. Agr. Food Chem. 9:301-304.

Whitaker, J.R. 1976. Development of flavor, odor, and pungency in onion and garlic. Adv. Food Res. 22;73-133. 